\title{
Learning from the missed opportunities for HIV testing
}

\section{Joanne D Stekler, ${ }^{1,2}$ Matthew R Golden ${ }^{1,2}$ EDITOR'S CHOICE}

In 2006, the US Centers for Disease Control and Prevention recommended expanding HIV testing in healthcare settings ${ }^{1}$ in order to identify the estimated $25 \%$ of HIV-infected persons who are unaware of their status and who may be responsible for up to $50 \%$ of HIV transmission. ${ }^{2}$ These recommendations partly attribute the failure of risk-based testing to issues related to access to care and the inability to target testing if individuals do not admit to potentially stigmatising risk behaviours or are unaware of their risk. However, in this issue of Sexually Transmitted Infections, Brown and colleagues $^{3}$ (see page 4) present data that suggest that the failure of targeted testing may be partly due to missed opportunities for HIV testing in readily identified highrisk populations.

Studying men who have sex with men (MSM) attending 15 genitourinary medicine (GUM) clinics in the UK between 1999 and 2002, the authors detected anti-HIV antibodies in 737 (6.7\%) of 10935 specimens obtained during visits when blood was obtained for syphilis testing but HIV testing did not occur (fig 1). These results are consistent with other published studies, including an anonymous serosurvey conducted at 28 US sexually transmitted disease (STD) clinics in 1997. In nearly all clinics HIV prevalence was 1.4 to 18 times higher among specimens from individuals who were not tested for HIV compared with specimens from individuals who were tested. ${ }^{4}$ These studies could overestimate the numbers of undiagnosed persons if multiple specimens were obtained from unique individuals during repeat visits or if infected individuals did not acknowledge their HIV status and refused testing. However, it is probably less important to estimate these numbers with precision than to acknowledge that testing is probably inadequate even in venues serving

\footnotetext{
${ }^{1}$ Department of Medicine and Center for AIDS and STD, University of Washington, Seattle, Washington, USA;

${ }^{2}$ Public Health, Seattle and King County, Seattle,

Washington, USA

Correspondence to: Dr J Stekler, Box 359931,

Harborview Medical Center, 325 Ninth Avenue, Seattle, WA 98104, USA; jstekler@u.washington.edu
}

high-risk individuals and to understand why testing was not offered or accepted.

For established testing programmes, these findings suggest two future objectives: (1) to increase the proportion of patients who are tested when they present for care and (2) to increase the frequency with which high-risk populations access care. The use of rapid antibody testing could increase the uptake of testing ${ }^{5}$ because patients may prefer rapid testing ${ }^{6}$ and more testers may receive test results ${ }^{78}$ compared with standard testing. Routinisation of testing through "optout" strategies could also increase HIV testing. Individuals attending UK clinics with opt-out testing policies had increased odds (odds ratio 1.59; 95\% CI 1.09 to $230, p=0.02$ ) of being offered an HIV test.' With opt-out strategies, one review estimated that $71-98 \%$ of women received an HIV test during pregnancy, compared with $25-83 \%$ in opt-in programmes. ${ }^{10}$ Additional procedural streamlining, by eliminating written consent requirements ${ }^{11}$ or delinking counselling and testing, could increase the uptake of HIV testing further.

Some individuals with a recent negative HIV test or risky exposure may be offered but defer testing because of concerns about the antibody-negative "window period". Are modifications needed to the recommendations to defer testing until 3 months after a potential exposure?
Newer antibody assays reduce the window period to 1 month, ${ }^{12}$ and pooled nucleic acid amplification testing programmes ${ }^{13}$ shorten it further. The avoidance of testing during primary HIV infection, when individuals are highly infectious, ${ }^{14}$ is likely to be counterproductive to goals of reducing HIV transmission. In fact, the phylogenetic analysis described by Brown et $a^{\beta}$ suggests that transmission from recently infected individuals may have occurred, and similar studies estimate that transmission during primary infection accounts for $25-49 \%$ of incidence. ${ }^{15-18}$ There is a need for more frequent HIV testing in high-risk populations, use of the most sensitive tests and consideration of education programmes designed to teach the symptoms of acute HIV infection. Although these strategies cannot eliminate transmission that occurs within days following HIV acquisition, ${ }^{12}$ they should decrease the time that HIV-infected individuals are unaware of their status and their potential for transmission.

Fortunately, Brown et $a l^{\beta}$ highlight a problem that is already being addressed. In UK GUM clinics in 2006, HIV testing was offered to $86 \%$ of MSM and testing occurred at $71 \%$ of visits. ${ }^{9}$ Similarly, the Centers for Disease Control and Prevention MSM Surveillance Monitoring Project reported that the proportion of MSM tested for HIV among four STD clinics increased from 49\% in 2002 to $61 \%$ in $2006 .{ }^{19}$ In our public health STD clinic in Seattle, MSM not known to be HIV infected received confidential testing during $84 \%$ of clinic visits in $2007-8$ and others received anonymous testing. We are moving in the right direction.

Our second goal for the future is to leave behind the traditional, passive approach of offering HIV testing to

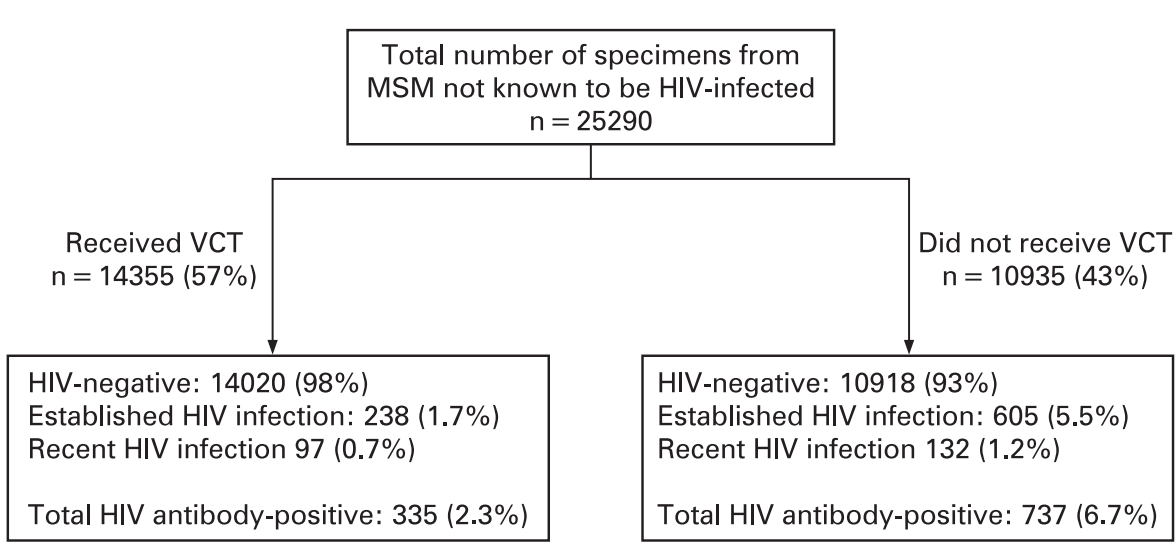

Figure 1 Proportion of specimens testing HIV antibody positive. MSM, men who have sex with men; VCT, voluntary confidential HIV testing. Data taken from Brown et al (see page 4). 
individuals who come to us. Modern technologies such as e-mail, text messaging and other online resources provide the potential for public health to play a more active role in increasing the frequency with which high-risk individuals seek testing. Although these technologies are not well studied to date, reminder and recall systems have been successfully employed to increase vaccination rates. ${ }^{20} 21$ In the interim, we look to mathematical modellers to inform us of the cost-effectiveness of prompting HIV testing and the ideal frequency of testing.

In the past decade, HIV testing and care services have expanded worldwide. In the UK and USA, more individuals attending GUM/STD clinics are receiving HIV testing and are receiving it more frequently. HIV testing remains one of the sharpest tools in the HIV prevention toolbox. Now we should wield that tool more efficiently. To do so, we should: (1) remove the remaining barriers to testing and move towards "opt-out" strategies; (2) advocate for the routine use of the most sensitive HIV tests, including pooled nucleic acid amplification testing in MSM and other groups with a high HIV incidence and (3) actively strive to increase the frequency of testing among those at highest risk of HIV acquisition. Programmatic research is required to develop and disseminate effective interventions and models to these ends. We still have much work to do to identify less accessible populations at risk of HIV infection, but as Brown et $a l^{3}$ make clear, we could do more to diagnose HIV in individuals identified as high risk who already access care.

Funding: This work was supported in part by grants from the National Institutes of Health (K23 Al65243) and University of Washington Center for AIDS Research New Investigator Award to JS (Al-27757).

Competing interests: None.

Accepted 9 December 2008

Sex Transm Infect 2009;85:2-3.

doi:10.1136/sti.2008.035329

\section{REFERENCES}

1. Branson BM, Handsfield HH, Lampe MA, et al. Revised recommendations for HIV testing of adults, adolescents, and pregnant women in health-care settings. MMWR Recomm Rep 2006;55:1-17; quiz CE11-14

2. Marks G, Crepaz N, Janssen RS. Estimating sexual transmission of HIV from persons aware and unaware that they are infected with the virus in the USA. AIDS 2006;20:1447-50.

3. Brown AE, Murphy G, Rinck G, et al. Implications for HIV testing policy derived from combining data on voluntary confidential testing with viral sequences and serological analyses. Sex Transm Inf 2009;85:4-9.

4. Weinstock H, Dale M, Linley L, et al. Unrecognized HIV infection among patients attending sexually transmitted disease clinics. Am J Public Health 2002;92:280-3.

5. Rietmeijer CA, Lloyd LV, Feuss B. Trends in HIV testing before and after the introduction of rapid testing in a busy urban sexually transmitted infection (STI) clinic. In: 43rd Annual Meeting of IDSA; 6-9 October 2005, San Francisco, IDSA. CA. [abstract \#875].

6. Spielberg F, Branson BM, Goldbaum GM, et al. Overcoming barriers to HIV testing: preferences for new strategies among clients of a needle exchange, a sexually transmitted disease clinic, and sex venues for men who have sex with men. J Acquir Immune Defic Syndr 2003;32:318-27.

7. Hutchinson $\mathbf{A B}$, Branson BM, Kim A, et al. A metaanalysis of the effectiveness of alternative HIV counseling and testing methods to increase knowledge of HIV status. AIDS 2006;20:1597-604.

8. Spielberg F, Branson BM, Goldbaum GM, et al. Choosing HIV counseling and testing strategies for outreach settings: a randomized trial. J Acquir Immune Defic Syndr 2005;38:348-55.
9. Munro HL, Lowndes CM, Daniels DG, et al. National study of HIV testing in men who have sex with men attending genitourinary clinics in the United Kingdom. Sex Transm Inf 2008;84:265-70.

10. HIV testing among pregnant women-United States and Canada, 1998-2001. MMWR Morb Mortal Wkly Rep 2002;51:1013-16.

11. Zetola NM, Grijalva CG, Gertler S, et al. Simplifying consent for HIV testing is associated with an increase in HIV testing and case detection in highest risk groups, San Francisco January 2003-June 2007. PLOS ONE 2008;3:e2591.

12. Stekler J, Maenza J, Stevens CE, et al. Screening for acute HIV infection: lessons learned. Clin Infect Dis 2007;44:459-61.

13. Stekler J, Swenson PD, Wood RW, et al. Targeted screening for primary HIV infection through pooled HIV-RNA testing in men who have sex with men. AIDS 2005;19:1323-5.

14. Wawer MJ, Gray RH, Sewankambo NK, et al. Rates of HIV-1 transmission per coital act, by stage of HIV-1 infection, in Rakai, Uganda. J Infect Dis 2005;191:1403-9.

15. Yerly S, Vora S, Rizzardi P, et al. Acute HIV infection: impact on the spread of HIV and transmission of drug resistance. AIDS 2001;15:2287-92.

16. Pao D, Fisher M, Hue S, et al. Transmission of HIV-1 during primary infection: relationship to sexual risk and sexually transmitted infections. AIDS 2005;19:85-90.

17. Brenner BG, Roger M, Routy JP, et al. High rates of forward transmission events after acute/early HIV-1 infection. J Infect Dis 2007;195:951-9.

18. Lewis F, Hughes GJ, Rambaut A, et al. Episodic sexual transmission of HIV revealed by molecular phylodynamics. PLoS Med 2008;5:e50.

19. Helms DJ, Weinstock HS, Mahle KC, et al. HIV testing frequency among MSM attending STD clinics: implications for HIV prevention and surveillance. $J$ Acquir Immune Defic Syndr 2008; in press.

20. Briss PA, Rodewald LE, Hinman AR, et al. Reviews of evidence regarding interventions to improve vaccination coverage in children, adolescents, and adults. The Task Force on Community Preventive Services. Am J Prev Med 2000;18(1 Suppl):97-140.

21. Jacobson VJ, Szilagyi P. Patient reminder and patient recall systems to improve immunization rates. Cochrane Database Syst Rev 2005; (3):CD003941. 